\title{
Overview of BES-GBS Project: The Role of Anisotropy on the Self- Organization of Gas Bubble Superlattice
}

\author{
Jian Gan, Yongfeng Zhang
}

October 2017

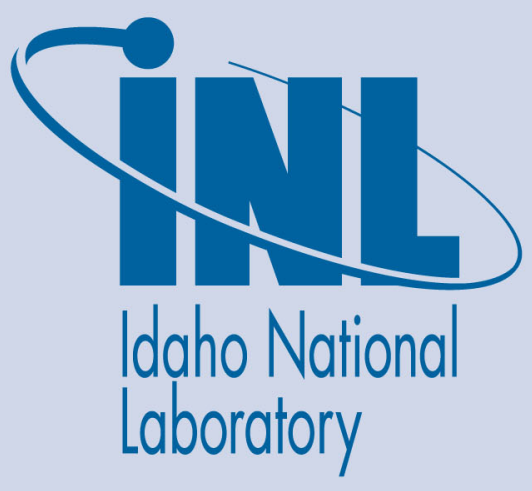

The INL is a U.S. Department of Energy National Laboratory operated by Battelle Energy Alliance 


\section{Overview of BES-GBS Project: The Role of Anisotropy on the Self-Organization of Gas Bubble Superlattice}

Jian Gan, Yongfeng Zhang

October 2017

Idaho National Laboratory Idaho Falls, Idaho 83415

http://www.inl.gov

Prepared for the

U.S. Department of Energy

Under DOE Idaho'Operations Office

Contract DE-AC07-05ID14517, DE-AC07-05ID14517 


\title{
Overview of BES-GBS Project:
}

The Role of Anisotropy on the Self-Organization of Gas Bubble Superlattice

\author{
Jian Gan \\ Idaho National Laboratory
}

September 4th, 2018

BES-GBS Project Review

Idaho National

Idaho Falls, Idaho 


\section{Acknowledgement}

\section{Contributors}

Yongfeng Zhang, Chao Jiang, Yipeng Gao, Daniel Schwen, Cheng Sun, Lingfeng He

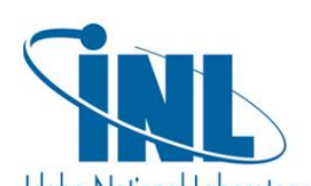

David Sprouster and Lynne Ecker

The work was supported by the U.S.

Department of Energy, Office of Science, Materials Sciences and Engineering Division under FWP C000-14-003.

The support from NSUF user project is acknowledged

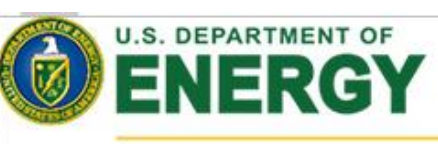

Office of Science

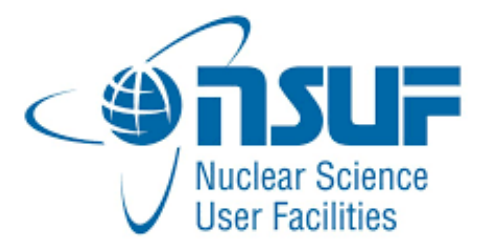

The guidance and advice from the BES program managers Linda Horton, John Vetrano and Jim Horwitz are much appreciated 


\section{Outline of Project Review Presentations}

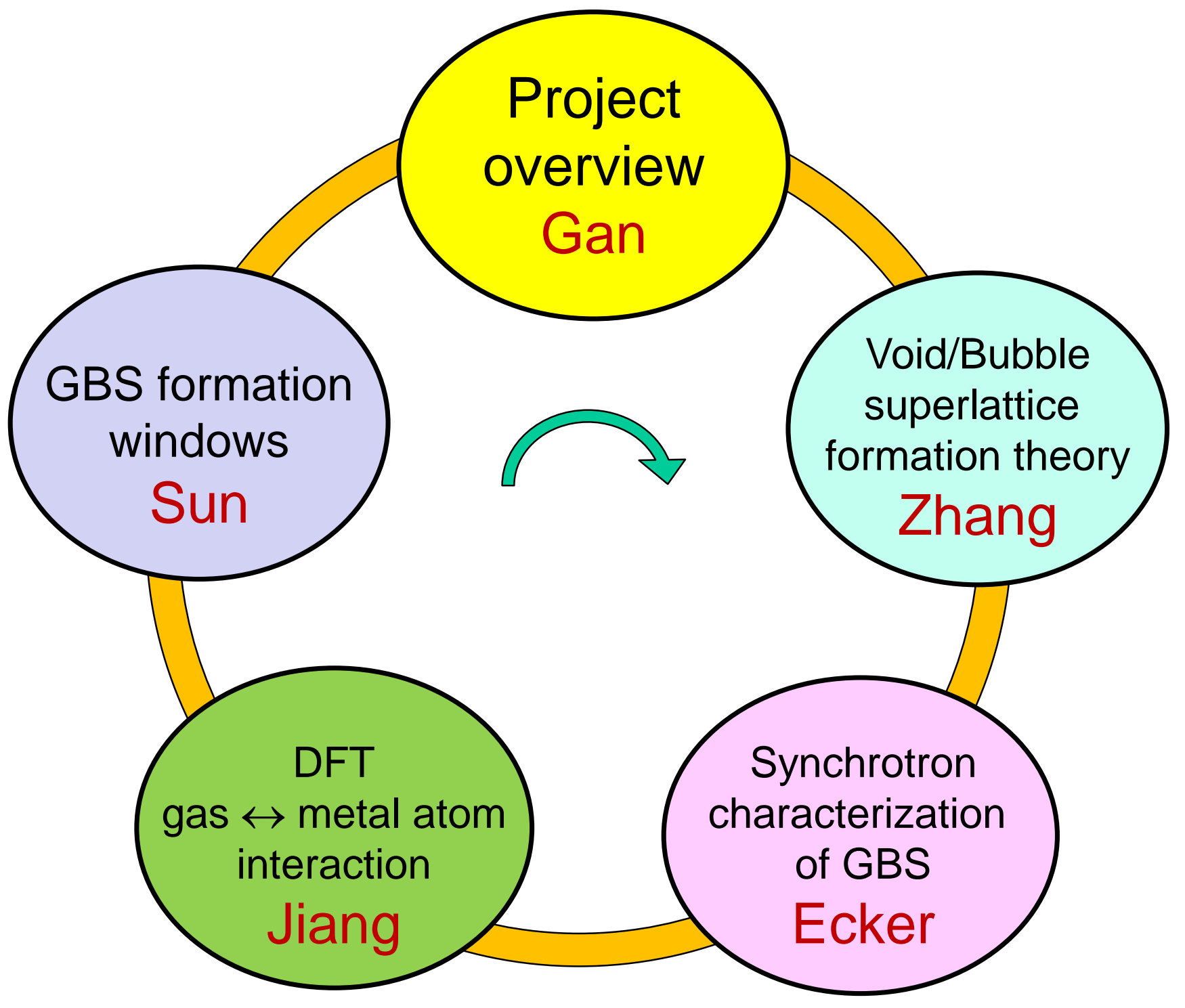




\section{Motivation and Broad Impact}

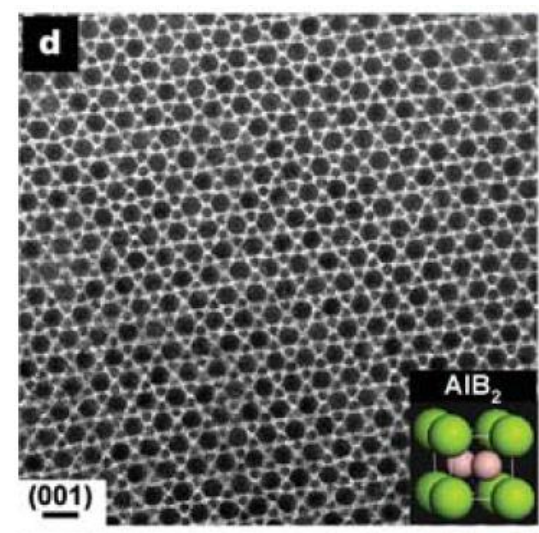

$\mathrm{PbS}-\mathrm{Pd}$ binary

nanoparticle superlattice

Shevchenko, Nature (2006)

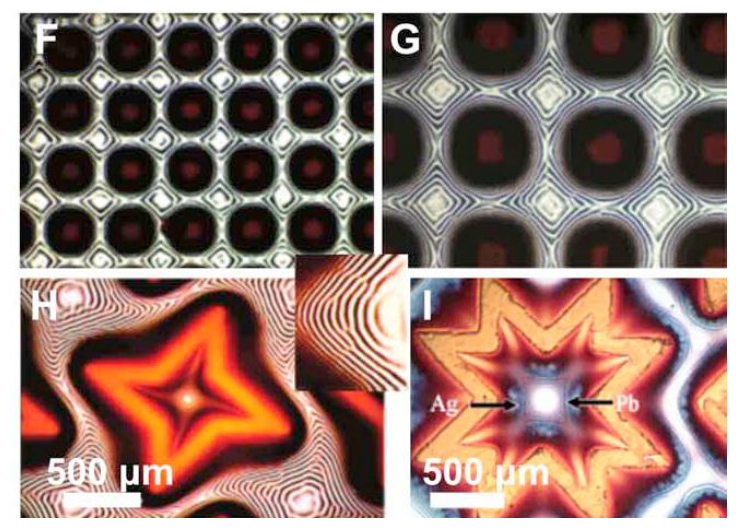

Liesegang patterns

(periodic precipitates) in diffusionreaction systems

Nakouzi, Science Advances (2016)

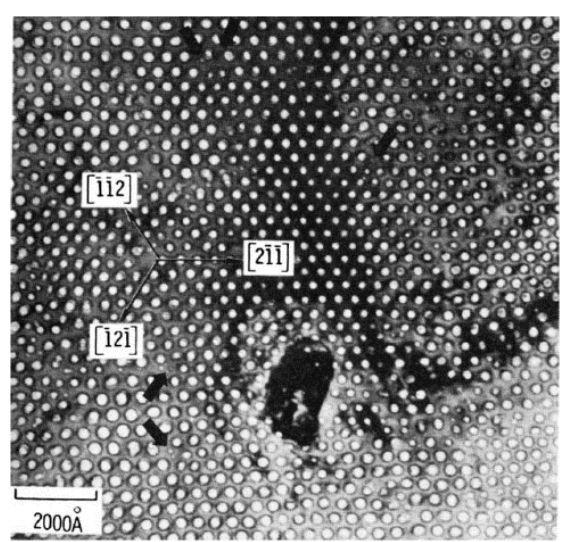

Void superlattice in $\mathrm{Nb}$ bulk

Ghoniem, JCAMD (2001)

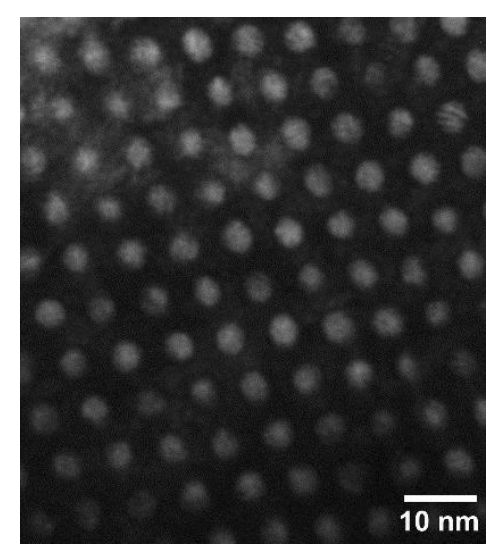

Xe gas bubble superlattice in irradiated U-7Mo

Gan, JNM (2012)

- Self-organization has been widely observed in far-from-equilibrium systems in the fields of physics, chemistry, and biology.

- It can be driven by thermodynamics (e.g., crystallization), dynamics (e.g., instability), or both.

- Understanding the underlying self-organization mechanisms may help us control the pattern formation through a "materials-by-design" approach, e.g., nanopatterned structures with novel electronic, magnetic, photovoltaic, or radiation-resistant properties.

- Radiation, although often associated with damage, can be a powerful tool to create these tailored microstructures provided the physics is understood. 


\section{Key Science Questions \& Hypothesis}

- What drives the formation of superlattices instead of random voids or bubbles?

Hypothesis:

The anisotropy on elasticity and/or diffusion is responsible for the self-organization of void/bubble superlattice.

- How does the irradiation condition in terms of temperature, flux and fluence, affect void/bubble superlattice formation and their properties?

Hypothesis:

The self-organization involves the interplay of thermodynamics and defect dynamics, both dependent on the irradiation conditions. Therefore, the superlattice properties like superlattice parameter can be manipulated by varying irradiation conditions.

- What determines the thermal stability of void or bubble superlattice?

Hypothesis:

Voids and bubbles in superlattice are thermally more stable than random voids and bubbles. But they are not thermodynamically stable and they can coarsen upon annealing. The coarsening kinetics depends on the ordering and the type of gas atoms. 


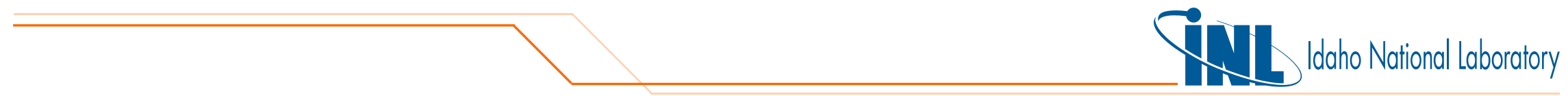

\section{Materials and Properties to address Hypothesis}

\begin{tabular}{|c|c|c|c|}
\hline $\begin{array}{l}\text { Property } \rightarrow \\
\text { Material } \downarrow\end{array}$ & Elastically & SIA Diffusion & Note \\
\hline $\mathrm{Fe}$ & anisotropic & isotropic & $\begin{array}{l}\text { 1-D diffusion for } \\
\text { small int. clusters }\end{array}$ \\
\hline W & isotropic & anisotropic & 1 -D along $<111>$ \\
\hline Mo & anisotropic & anisotropic & $1-D$ \\
\hline $\begin{array}{c}W(x) \\
x=R e \text { or } F e\end{array}$ & isotropic & isotropic & $\begin{array}{l}\text { To be acquired } \\
\text { or fabricated }\end{array}$ \\
\hline \multicolumn{4}{|c|}{ Inert Gas Atom Size } \\
\hline
\end{tabular}




\section{Integrated Research Approach}

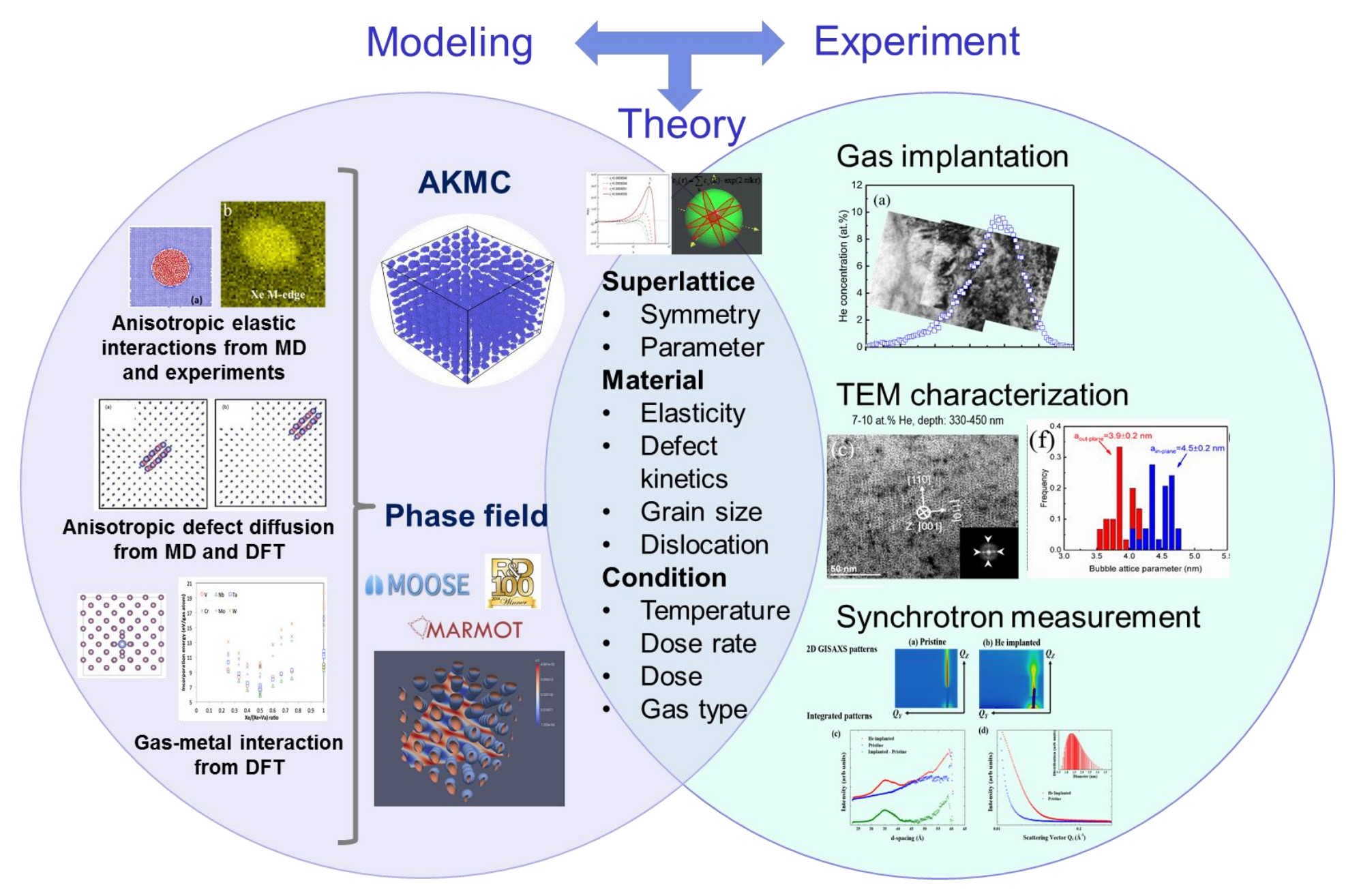




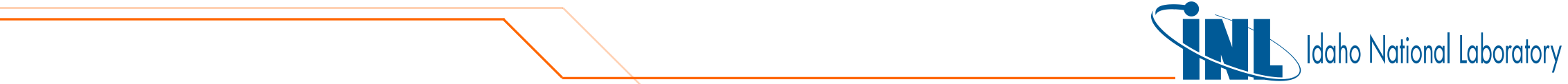

\section{BES Project Team (INL and BNL)}

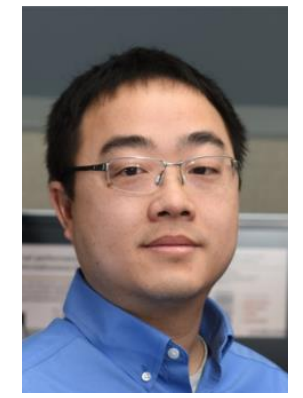

Yongfeng Zhang

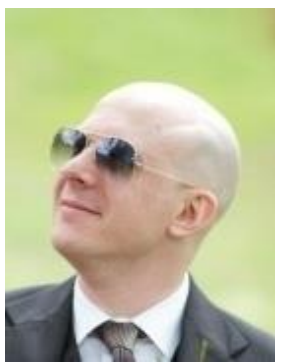

David

Sprouster

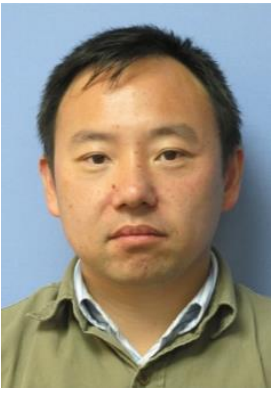

Chao Jiang

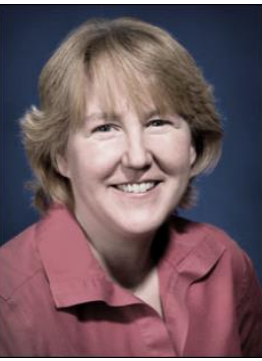

Lynne

Ecker

- $\$ 700 \mathrm{k} / \mathrm{yr}^{\star}+\$ 300 \mathrm{k}$ seed.

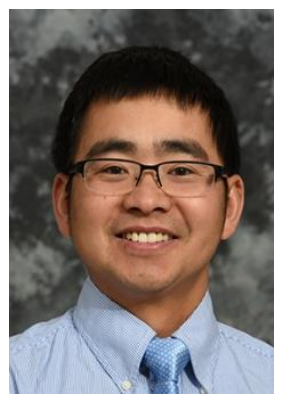

Lingfeng $\mathrm{He}$

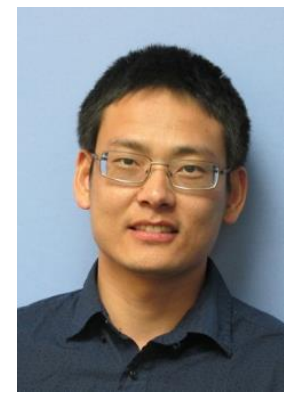

Cheng Sun

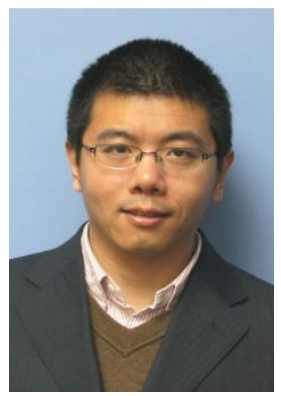

Yipeng Gao

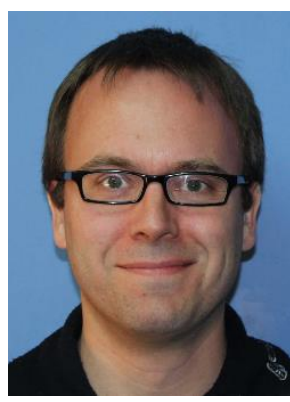

Daniel Schwen

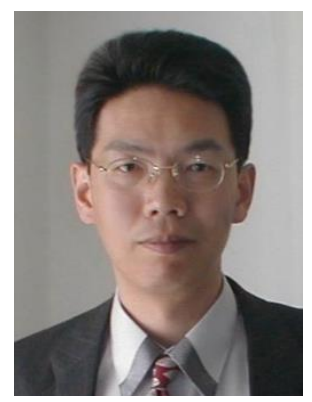

Jian

Gan

- This project is crucial to promote science

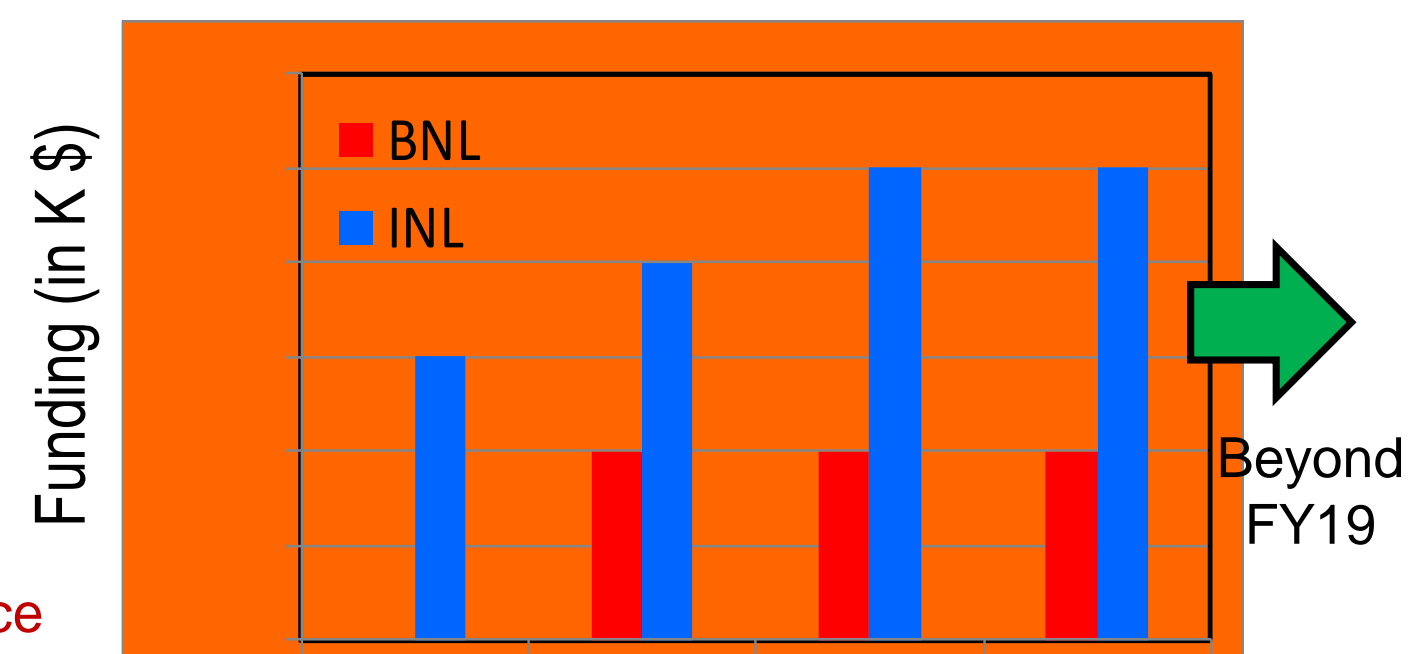
and fundamental research at INL.

- This BES core project has a large impact. 


\section{The Synergies between INL and BNL}
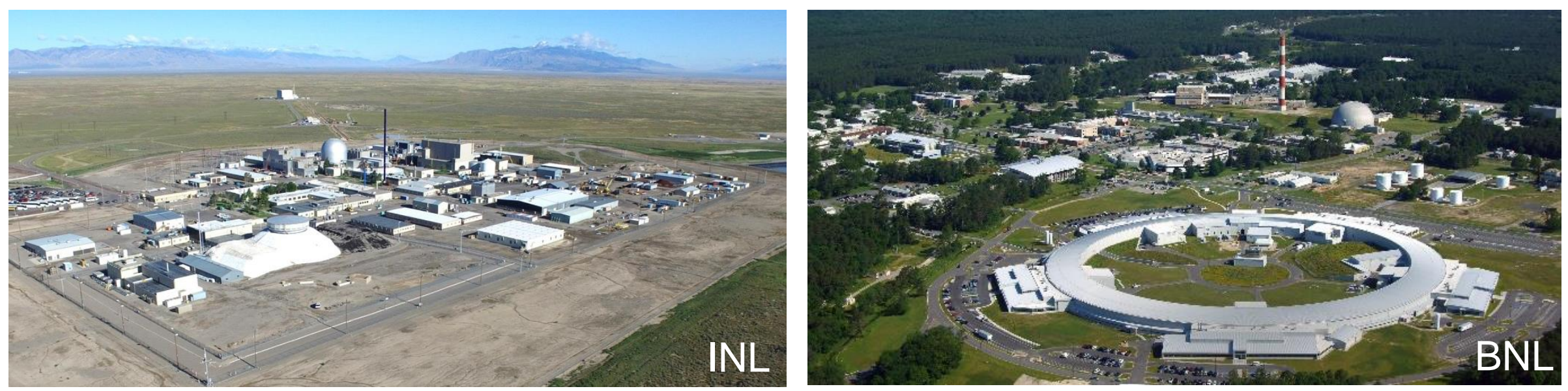

- Joint effort on proposal development.

- Complementary and unique strength, experience and capabilities.

- INL leads effort on irradiation, TEM characterization and modeling.

- BNL leads effort on synchrotron x-ray characterization.

- Bi-weekly BlueJean teleconf promotes new ideas, coordinated research, joint publication and in-depth discussion on the work in progress.

- Synergy on this project promotes joint research proposal between INL-BNL in other research areas. 


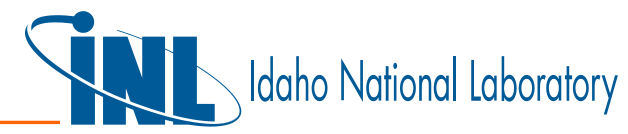
BES \& NE User Facilities are Critical to Facilitate Synergies

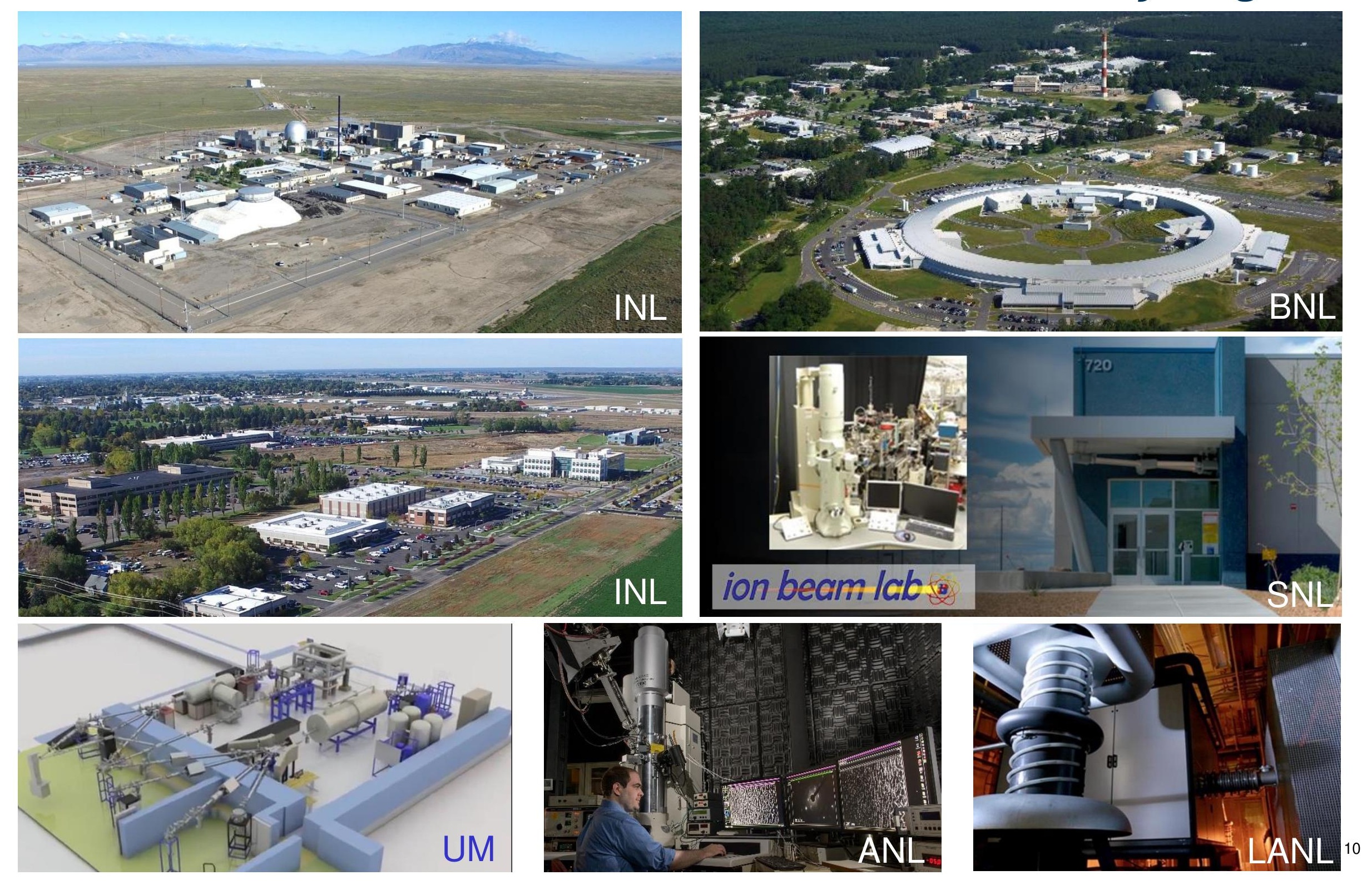




\section{然}

\section{User Proposals Awarded (Led by INL, 6)}

- C. Sun, L. He, J. Gan, "Nanopatterning of Ne/Ar gas-bubble superlattice in bcc metals", The Center for Integrated Nanotechnologies (CINT), Basic Energy Science User Facility, 2018-2019.

- C. Sun, L. He, Y. Zhang, J. Gan, "Nanopatterning of gas-bubble superlattice in bcc metals", The Center for Integrated Nanotechnologies (CINT), Basic Energy Science User Facility, 2017-2018.

- J. Gan, L. He, C. Sun, "Investigation of gas bubble behavior in metals using in-situ Ne, Ar and Kr ion irradiation", Intermediate Voltage Electron Microscope (IVEM), Nuclear Science User Facility (NSUFRTE), 2017-2018.

- C. Sun, L. He, Y. Zhang, J. Gan, "The window of gas-bubble superlattice formation in bcc metals" Michigan Ion Beam Laboratory (MIBL), Nuclear Science User Facilities (NSUF-RTE), 2016-2017.

- L. He, C. Sun, J. Gan, "Self-Organization of Gas-Bubble Superlattice in Selected Metals", Intermediate Voltage Electron Microscope (IVEM), Nuclear Science User Facility (NSUF-RTE), 2016-2017.

\section{Pending:}

- C. Sun, D. J. Sprouster, L. He, J. Gan, "Self-organization of noble gas bubbles in nuclear fuels and materials under ion implantation" Michigan Ion Beam Laboratory (MIBL), Nuclear Science User Facilities (NSUF-RTE), 2018-2019. 


\section{User Proposals Awarded (Led by BNL, 5)}

- D. J. Sprouster, L. Ecker, S. Gill, "Gas bubble superlattice formation in metals", The Centre for Functional Nanomaterials (CFN), Basic Energy Science User Facility, 2018-2020.

- D. J. Sprouster, L. Ecker, C. Sun, C. Jiang, "XAS of ultra-dilute inert gas atoms in BCC metals", Inner Shell Spectroscopy (ISS) beamtime proposal, National Synchrotron Light Source-II, Basic Energy Science User Facility, 2018-2019.

- D. J. Sprouster, L Ecker, C. Sun, J. Gan, "Characterizing self-organization due to ion beam radiation damage" Life Science X-ray Scattering (LIX) beamtime proposal, National Synchrotron Light Source-II, Basic Energy Science User Facility, 2018-2019.

- L. Ecker, S. Gill, D.J. Sprouster, "Utilizing Hard X-Ray Nanoprobe to understand the role of Anisotropy on the Self-Organization of Gas Bubble Superlattices", Hard X-ray Nanoprobe (HXN) beamtime proposal, National Synchrotron Light Source-II, Basic Energy Science User Facility, 2017-2018.

- D. J. Sprouster, .J Gan, L Ecker, "Location and Structure Determination of Fission Bubbles in Nuclear Fuel", Submicron Resolution X-ray Spectroscopy (SRX) beamtime proposal, National Synchrotron Light Source-II, Basic Energy Science User Facility, 2017-2018. 


\section{Publication Status}

\section{Published (5)}

- C. Sun, D. Sprouster, K. Hatter, L.E. Ecker, L. He, Y. Gao, Y. Zhang, J. Gan, "Formation of tetragonal gas bubble superlattice in bulk molybdenum under helium ion implantation", Scripta Materialia, 149 (2018) 26-30.

- C. Jiang, Y. Zhang, Y. Gao, J. Gan. “Ab initio theory of noble gas atoms in bcc transition metals”, Phys. Chem. Chem. Phys., 20 (2018) 17048.

- Y. Gao, Y. Zhang, D. Schwen, C. Jiang, C. Sun, J. Gan \& X. Bai, "Theoretical prediction and atomic kinetic Monte Carlo simulations of void superlattice self-organization under irradiation", Scientific Report (2018) 8:6629.

- J. Gan, C. Sun, L. He, Y. Zhang, C. Jiang, Y. Gao. "Thermal stability of helium bubble superlattice under TEM in-situ heating”, J. Nucl. Mater., 505 (2018) 207-211.

- Y. Gao, Y. Zhang, C. Jiang, J. Gan \& D. Schwen, "Formation and Self-organization of Void Superlattice under Irradiation: a Phase Field Study", Materialia, 1 (2018) 78-88.

\section{Submitted (2)}

- D.J. Sprouster, C. Sun, Y. Zhang, S.N. Chodankar, J. Gan, L.E. Ecker, "Irradiationdependent Helium Gas Bubble Superlattice in Tungsten", submitted to Scientific Report, working on revision to address reviewer's comments.

- Y. Zhang, Y. Gao, C. Sun, D. Schwen, C. Jiang, J. Gan,, "The role of kinetic anisotropy in void superlattice self-organization", working on revision for submission. 


\section{Presentations (2 meetings, 3 conf., 3 seminar)}

- C. Sun, et al (poster), "Self-organization of gas bubble superlattice in nuclear fuels and materials", NS\&T Strategic Advisory Committee Meeting, Idaho Falls, May, 2018.

- L. He, et al. (poster), "Advanced microstructural characterization of nuclear fuels", NS\&T Strategic Advisory Committee Meeting, Idaho Falls, May, 2018

- Y. Gao, et al. (speaker), "A phase field study of void superlattice formation in irradiated materials", TMS 2018 annual meeting, Phoenix AZ, March, 2018.

- D. Sprouster, et al. (speaker), "Self-Organization of Gas Bubble Superlattices", TMS 2018, Phoenix AZ, March, 2018.

- Y. Zhang, et al, "Void Superlattice Formation: Symmetry and Lattice Parameter Selection", TMS 2018, Phoenix, AZ, March 2018.

- Y. Zhang, et al, "Self-organization of nanoscale void superlattice under radiation", Student Seminar at the Oregon State University, November 2017.

- Y. Zhang, et al, "Rate theory formulation of void superlattice self-organization", Guest lecture in a graduate course, MIT, November 2017.

- Y. Zhang, et al, "Self-organization of nanoscale void superlattice by chemical freezing of phase separation", Student Seminar at the North Carolina State University, December 2018. 


\section{Significant Outcomes}
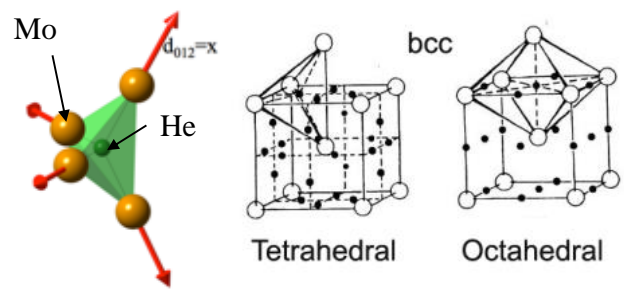

Tetrahedral Octahedral

Much better correlation with shear

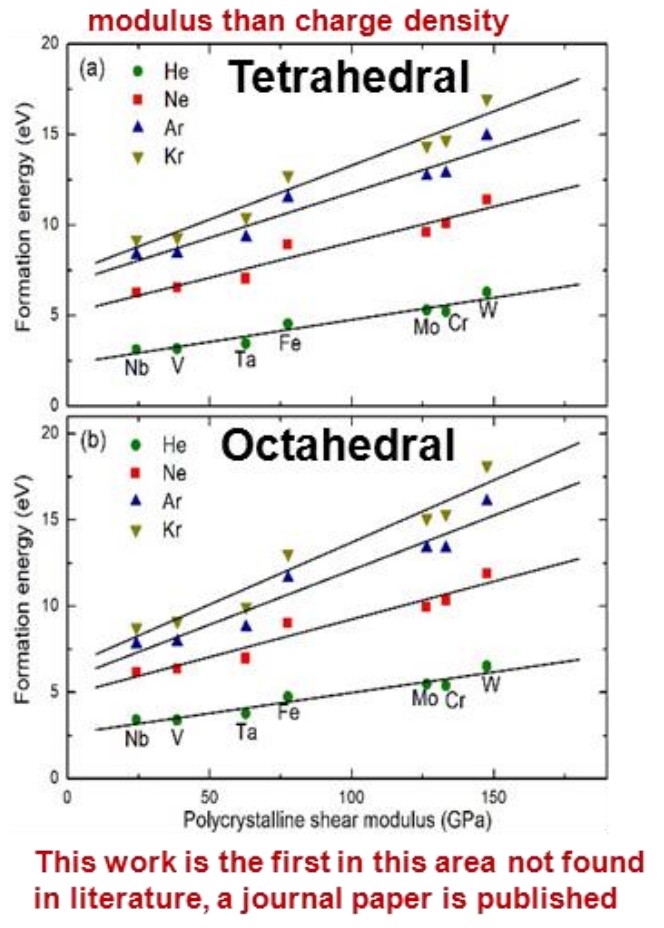

This work is the first in this area not found in literature, a journal paper is published
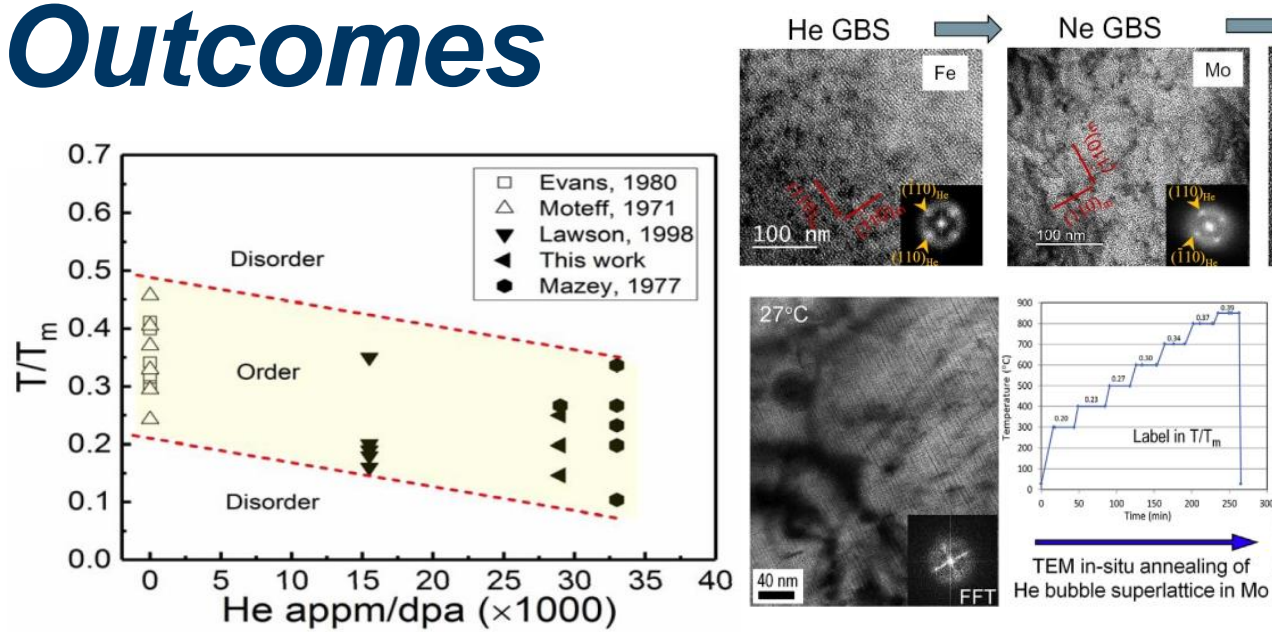

He bubble superlattice in Mo 40 n

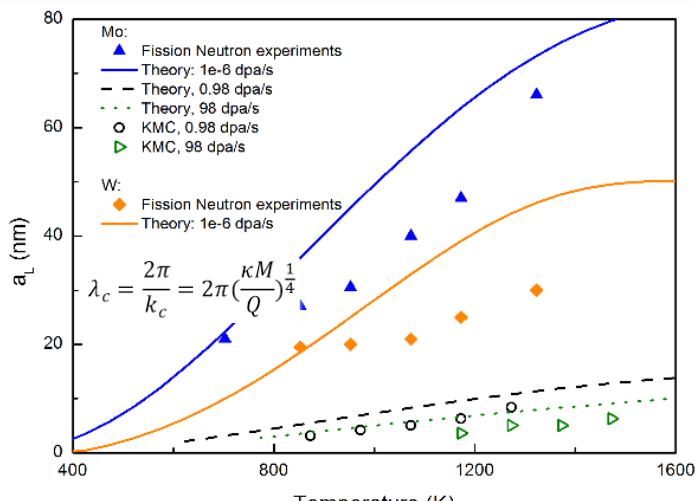

Temperature (K)

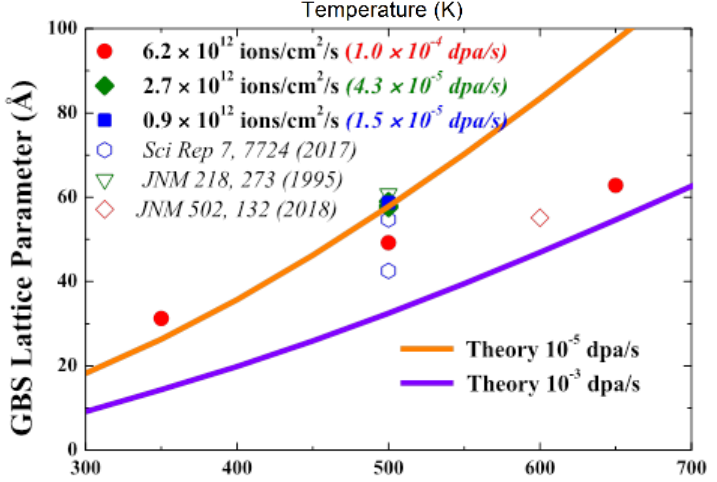

Implantation Temperature $\left({ }^{\circ} \mathrm{C}\right)$
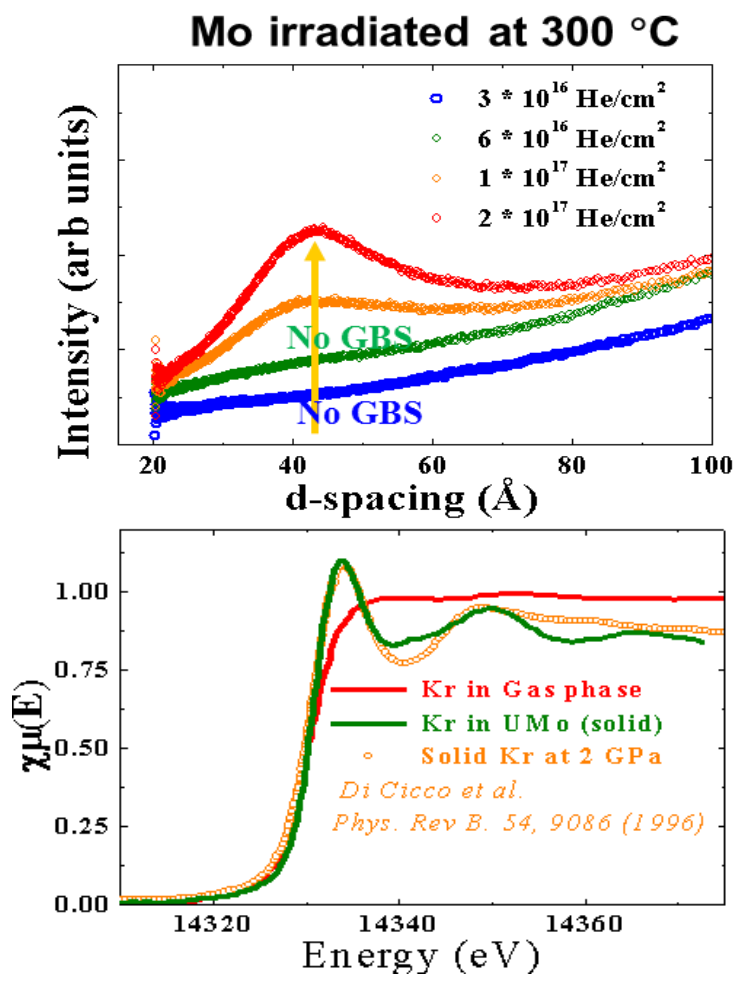

Ex-situ heating result agrees with in-situ heating result. Coarsening leads to $\mathrm{He}$ GBS disorder at $1000^{\circ} \mathrm{C} / 60 \mathrm{~min}$ 


\section{What have we learned so far?}

(1) What define the lattice parameter and structure of void superlattice? A complete rate theory formulation was developed which can now predict both lattice parameter and structure. The predictions are consistent with experiments and simulations with regards to the effects of temperature, flux and fluence (Zhang's talk).

(2) How radiation conditions in terms of temperature, flux and fluence affect gas bubble superlattice characters: lattice parameter and structure? Radiation conditions for He GBS formation have been successfully established experimentally, with TEM analysis and nondestructive and high-resolution synchrotron X-ray techniques to determine lattice parameter and structure, and lattice strain and pressure in GBS (Ecker \& Sun's talk).

(3) How gas atom interact with lattice atoms? A unified model with four input parameters trained by first principle calculations is developed to describe the incorporation energy of various inert gases in many bcc metals. (Jiang's talk)

\section{What we will answer in a short future}

(1) Are void and gas bubble superlattice (with various inert gases) fundamentally different? They share the same lattice structure; the theoretical predictions on void superlattice seem applicable to gas superlattice as well; their formation processes can be similar. These suggest void and gas superlattices may be explained using a unified theory, particularly when elastic strain energy is not important.

(2) What is the effect of gas bubble pressure? Over-pressurized bubbles could cause different behavior, particularly elastic strain energy. This will be studied in future work.

(3) Is effect of elasticity anisotropy important for large gas atoms? This will be studied in future work. 


\section{Other Important Questions}

- Why is $\mathrm{Kr}$ and $\mathrm{Xe}$ GBS so difficult to form under ion irradiation?

- Why does Xe GBS in U-Mo have fcc structure on bcc host metal?

- Why Xe GBS is much better ordered than that of He GBS?

- Why void superlattice is in general much better ordered than that of He GBS?

- Why void thermal stability in void superlattice in Mo is much higher than that of isolated voids $\left(1500{ }^{\circ} \mathrm{C}\right.$ vs. $\left.1100{ }^{\circ} \mathrm{C}\right)$ ?

- Why Xe GBS thermal stability is much higher than He GBS $\left(0.78 \mathrm{~T}_{\mathrm{m}}\right.$ vs. $\left.0.44 \mathrm{~T}_{\mathrm{m}}\right)$ ? 


\section{SUMMARY}

- A rate theory based instability analysis has been developed which can predict both lattice parameter and structure of void superlattice without using any fitting parameters. The theoretic predictions are consistent with both experiments and atomistic simulations.

- DFT calculation on inert gas atom interaction with bcc metal atoms generated important insight to help understanding the GBS formation mechanism and thermal stability.

- Many similarities have been observed between gas bubble and void superlattices, suggesting that they may form by the similar mechanisms, particularly in situations without substantial contribution from elastic strain energy.

- The synergies between TEM and Synchrotron analysis, and the experimental and modeling are critical to this BES project.

- We are facing the big challenges to close the knowledge gap on

(1) creating $\mathrm{Kr}$ or Xe GBS through ion irradiation and

(2) fully understanding its exceptional thermal stability

3) explaining the exceptional structural relationship (fcc on bcc).

This is a critical step and it has never been done before. The predictions from modeling will be used to optimize the experimental conditions. 